\title{
Risk approaches for delivering disaster relief supplies
}

\author{
Pamela C. Nolz • Frédéric Semet • Karl F. Doerner
}

Published online: 2 June 2011

(C) The Author(s) 2011. This article is published with open access at Springerlink.com

\begin{abstract}
We consider the problem of designing the logistic system to assure adequate distribution of relief aid in a post-natural-disaster situation, when damages to infrastructure may disrupt the delivery of relief aid. The problem is formulated as a multi-objective optimization problem, encompassing three objective functions of central interest in such problems. The first objective function is a measure of risk (various forms of such risk are analyzed). The second objective function measures the coverage provided by the logistic system in the distribution of relief aid to disaster victims. The third objective function represents total travel time. We focus on the risk of delivery tours for relief supplies, where risk here captures the threat that potential tours become impassable after the natural hazard event. In order to cope with a range of different natural disasters and policy objectives, we develop five approaches emphasizing
\end{abstract}

\footnotetext{
P. C. Nolz

Ecole des Mines de Saint-Etienne, CMP Georges Charpak, 13541 Gardanne, France e-mail:nolz@emse.fr

P. C. Nolz · K. F. Doerner

Department of Business Administration, University of Vienna, Bruenner Strasse 72, 1210 Vienna, Austria

F. Semet

Ecole Centrale de Lille, LAGIS, Cité Scientifique, 59651 Villeneuve d'Ascq, France e-mail: frederic.semet@ec-lille.fr

K. F. Doerner $(\varangle)$

Institute of Production and Logistics Management, Johannes Kepler University Linz, Altenberger Strasse 69, 4040 Linz, Austria e-mail: karl.doerner@jku.at
} 
different measures of tour-dependent risk. To cover both earthquake and flood risks, we consider correlated as well as uncorrelated risk measures. We develop a two-phase solution approach to reflect the dictates of real-world disaster relief motivating this analysis. The first phase generates potentially Pareto-optimal solutions to the overall multi-objective logistic design problem with respect to three objectives. For any given risk measure, the first-phase design problem is formulated as a multi-objective integer program and a memetic algorithm is proposed as the solution approach. The second phase is an enrichment procedure to generate a broader range of potentially Pareto-optimal alternatives. The suggested approach is tested on real-world data from the province of Manabí in Ecuador and the results associated with the different risk measures are analyzed to illustrate the value of the proposed approach for the design of disaster relief networks.

Keywords Multi-objective optimization · Disaster operations planning · Disaster risk

\section{Introduction}

\subsection{Motivation}

Natural disasters occur where natural hazards and vulnerable conditions converge. Human vulnerability to natural disasters is linked to a country's level of development and environmental quality (Peduzzi et al. 2009). Poor and socially disadvantaged groups in developing countries are particularly impacted by a disaster. As their possibilities to adapt to the post-disaster situation or recover from possible damages are limited, relief aid is of particular importance. In the context of a disaster, characterized by instability and the immediate need of help, high-quality decisions have to be made quickly to take the most appropriate measures. Many operations have to be carried out simultaneously, which requires intensive coordination plans.

In the classical four phases of emergency management (mitigation, preparedness, response, and recovery), the model presented in this paper belongs to the preparedness as well as the response phase. It encompasses several of the typical activities of these phases as listed in the survey paper of Altay and Green (2006) on disaster operations management. Van Wassenhove (2006) states that effective disaster management combines disaster preparedness and disaster response. The algorithm presented in this paper works with information, that is available before a disaster, such as road network data and number of inhabitants. Geodata can be assessed via satellite images and is available for almost all disaster-prone regions in electronic format, referring to disaster preparedness. By analyzing the topography and the road network of a concerned region, it is possible to estimate the risk values of road sections. Therefore, this information has to be assessed a priori and be maintained regularly. When a disaster strikes, information on the geographic extension of the affected region is added as well as statistical data on the disaster risks. Within a few minutes, solutions are provided on where to place water tanks and which roads to use for the transport of drinking water in the disaster response. This enables decision makers to have a detailed look at competing solutions and choose one that best fits their preferences. 
In this paper, we focus on the aspect of risk on delivery tours for disaster relief supplies. Risk is considered within a multi-objective problem formulation, in addition to a weighted mean of a minisum facility location criterion and a maximal covering location criterion on the one hand, and travel time on the other hand. Although the approaches in this paper can be easily adapted to the distribution of food or shelter, we exemplify their application on drinking water for several reasons. Water is an irreplaceable good that is essential to life. In case of an emergency, it is one of the first necessities urgently required. Moreover, clean water is obligatory for sanitary requirements. The distribution of drinking water plays a major role in disaster relief, where it is essential to provide clean water to the vulnerable population. If the affected people do not receive drinking water immediately, they are forced to use raw water from any available source, risking illnesses and epidemics. As opposed to medicine, water is directly delivered to the population that has non-restrictive access to the water tanks. Medicine, on the contrary, cannot be provided without restriction in order to prevent misuse and abuse. Unlike food or shelter-related items, water can easily be portioned to be distributed and is needed at a higher frequency.

In a post-disaster situation, characterized by high instability, it is important for the affected population to rely on the regular delivery of water. This concerns not only the time of the delivery, but also the locations where drinking water is made available. Receiving water the same day of the week and at the same time brings regularity and security into the lives of the affected population. Therefore, it is crucial to plan the delivery tours for drinking water such that they remain accessible in case of aftershocks or increasing water levels after floods.

Depending on the resources in the affected region, water is taken from an available source, for example, a well or a river. The raw water is cleaned in a drinking water treatment plant (located at the depot) and then loaded onto vehicles to be distributed. For our investigation, several locations are determined, where tanks and so-called 'tap stands' can be placed, which enable the surrounding population to get a certain amount of water when they need it. Each location represents a small geographical area and the population that needs to be supplied with drinking water, by a single point. These population center points are candidate locations for water tanks. Via a road network still available after the disaster, population center nodes can be visited but no node has to be visited. A population center node is covered if the people associated with this node can reach a water tank within a predefined travel time (for example $2 \mathrm{~h}$ ). Note that each population center is assigned to the nearest water tank, even if it is not covered by this tank (meaning that the population members have to walk longer than $2 \mathrm{~h}$ to get water).

Risk means the possibility that delivery tours become impassable after natural disasters. Bridges can easily be destroyed in case of an aftershock, roads adjacent to a river are at risk to be flooded. As a consequence, they could no longer be used and the delivery tours and possibly also the locations for water tanks could not be maintained. These critical arcs can be seen as bottlenecks, which determine the risk of a path. By avoiding these arcs, the risk of the whole path can be decreased.

The contribution of this paper is threefold. First, we develop and apply five approaches to evaluate the risk of delivery tours for disaster relief supplies to become impassable. To cover different types of disaster risk, we consider correlated as well as 
uncorrelated measures. Each risk approach is included alternatively in a multi-objective Covering Tour Problem (CTP) as an objective function. The CTP (Gendreau et al. 1997) consists of determining a minimum length tour over a subset of nodes, starting and ending at a depot, such that a subset of nodes is visited and a subset of nodes is covered by the tour. Coverage means that a node lies within a maximum distance from a node visited in the tour. Second, an extension of a Memetic Algorithm (MA) introduced in Nolz et al. (2009) is developed. Finally, it is shown that solution quality can be improved by a 2-phase solution method, contributing good compromise solutions for the potentially Pareto-optimal frontier.

\subsection{Literature review}

Logistics is a critical topic in humanitarian aid operations. Academic research in humanitarian logistics, especially in the field of Operations Research (OR), is limited but has evolved a lot in the past years. For two recent and relevant surveys, see Kovács and Spens (2007) and Altay and Green (2006).

However, only few relevant papers in the area of disaster relief have been published that focus on the last mile distribution of critical items to disaster victims. Hodgson et al. (1998) consider an accessibility problem in a developing country. They propose a covering tour model for planning mobile health care facilities in Ghana, where they take multiple classes of roads into account depending on their accessibility influenced by different weather types. Doerner et al. (2007) develop solution procedures for a combination of a location and a routing problem for mobile health care facilities in Senegal. Balcik et al. (2008) examine the allocation and distribution of emergency relief supplies from local distribution centers to demand locations, minimizing transportation costs and maximizing the benefit for aid recipients. Campbell et al. (2008) formulate two different objective functions for the routing of vehicles carrying critical items in a disaster relief application and study the corresponding models. Viswanath and Peeta (2003) formulate a multicommodity maximal covering network design problem with two objectives. They try to minimize routing costs, while maximizing the total demand covered. Budget constraints are introduced for the retrofitting of bridges destroyed by an earthquake. A pick-up and delivery problem for emergency situations is studied by Özdamar et al. (2004), where supply is limited and its availability varies over the planning horizon. The transportation problem is solved repetitively during the phase of aid deliveries, where the objective is to minimize the delay of arrivals at aid centers. A similar goal is optimized in Yi and Özdamar (2007), where a two-stage location-routing problem is solved in order to coordinate transportation of commodities as well as evacuation of wounded people. In De Angelis et al. (2007), weekly schedules of emergency deliveries by plane in Angola are developed for the World Food Programme, aimed at maximizing the total satisfied demand. Barbarosoglu et al. (2002) focus on scheduling helicopter activities in a disaster relief operation. They decompose the multiobjective problem according to tactical and operational decisions, allowing an interactive decision making process.

In the humanitarian context, the problem of finding survivable networks has not been investigated a lot. McLachlin et al. (2009) compare techniques and practices 
developed for uninterrupted for-profit supply chains to the not-for-profit interrupted humanitarian relief context. Kovács and Tatham (2009) examine the resource configurations of humanitarian versus military organizations in order to be able to respond to large-scale disruptions. Matisziw and Murray (2009) formulate a model to evaluate the disruption of arcs vital to network operations and apply it to the Ohio interstate highway system to support the assessment of disaster vulnerability.

Several related papers can be found in the area of telecommunications. Clímaco and Pascoal (2009) introduce a method to compute bi-objective shortest pairs, each including two disjoint simple paths. Gouveia et al. (2008) guarantee survivability of a network by determining $D$ node-disjoint paths. Soni and Pirkul (2002) design a communication network, where at least one communication route remains present between all pairs of nodes. Fortz (2000) defines several survivability levels for the network, depending on the importance of the nodes. This means that, for each level of survivability a specified number of paths between two nodes is required.

However, in case of a natural disaster, it is not adequate to search for disjoint paths or nodes. It is also not desirable to maintain one traversable route between all pairs of nodes, without assessing its risk. We rather want to avoid critical arcs on our path, such as bridges or low-lying roads. Therefore, instead of applying already existing techniques, it is necessary to develop approaches that consider the catastrophe hazard values of the arcs in the network. In order to model the risk of different types of natural disasters, approaches are developed that either consider correlated catastrophe hazards of the arcs in the network, or assume independence of the hazard values. In the case of an earthquake, the risk of a path to be disrupted depends on the independent threats of bridges to be destroyed by an aftershock. Bridges are on average located with some spatial distance in between and allow to use safe road sections (arcs) before crossing the next bridge. With respect to floods, correlated risk measures are more adequate, assuming that whole areas located along a river are flooded in consequence of rising water levels.

The remainder of the paper is organized as follows: In the next section, multiobjective decision making is introduced and the different risk approaches are presented as one of three objectives. In Sect. 3, the problem is described in detail. In Sect. 4, the solution procedure is introduced and explained. Computational experiments are presented in Sect. 5. The algorithm is tested on real-world data from the province of Manabí in Ecuador, and the different risk approaches are analyzed in order to evaluate the most appropriate risk measure for delivering disaster relief supplies. Finally, some concluding remarks and perspectives are provided.

\section{Multi-objective decision making}

\subsection{Motivation}

In a real-world context, especially in the field of disaster relief, several conflicting objectives have to be optimized. It is not possible to encompass all relevant criteria into a single objective function. For example, in disaster relief, monetary and nonmonetary objectives (e.g. costs and human lives) have to be differentiated. 
In general, multi-objective problems can be treated with two different approaches. On the one hand, all criteria can be weighted and combined into a single sum. Therefore, the preferences of the decision makers on the conflicting objectives are needed beforehand, making the decision process more difficult. On the other hand, a Pareto set approximation can be generated, enabling the decision makers to choose out of a set of diverse solutions according to their preferences.

\subsection{Solution concepts}

Because of the multi-objective nature of our problem, it is not possible to provide the decision maker with a single " optimal" solution, but rather a set of alternative solutions. However, by filtering out dominated solutions, the choice can be restricted to a small number of promising candidate solutions. The following definitions make this consideration precise:

Concept of dominance. A solution $x$ dominates a solution $x^{\prime}$ if $x$ is at least equally good as $x^{\prime}$ with respect to all objective functions, and better than $x^{\prime}$ with respect to at least one objective function.

Concept of Pareto efficiency. A solution $x^{*}$ is called Pareto-optimal (or nondominated) if there is no feasible solution that dominates $x^{*}$. The set of all nondominated solutions is referred to as the Pareto frontier (or nondominated frontier).

\subsection{Objectives}

We consider a multi-objective CTP, which consists of developing a set of vehicle routes with respect to three criteria: (1) a risk criterion, (2) a combination of the minisum facility location criterion (short: minisum criterion), which minimizes the sum of distances between all inhabitants of population center nodes and their nearest facility, and the maximal covering location criterion (MCLC), which minimizes the number of population members unable to reach a facility within a predefined maximum distance, and (3) a tour length criterion, measured in terms of travel time.

\subsubsection{Risk}

In a disaster relief operation, it is important to provide the affected population with water on a regular basis. This concerns not only the time of the delivery but also the locations, where drinking water is made available to the population.

In a post-disaster situation, delivery tours are exposed to the risk of being interrupted as a consequence of the previous disaster. For example, a given tour might no longer be usable if it contains a bridge that is destroyed by an aftershock of an earthquake. In order to cope with this risk and take it into consideration in our multiobjective problem formulation, we investigate three measures assuming independent catastrophe hazards of the traversable arcs: Number of alternative paths, unreachability and threshold risk. With respect to floods, correlated catastrophe hazard values are 

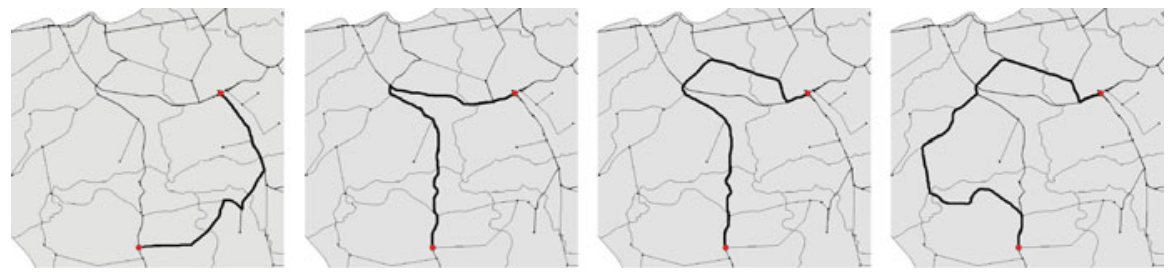

Fig. 1 Four alternative (R-T)-Pareto-optimal connections between two population nodes

Table 1 Notation

\begin{tabular}{ll}
\hline$J$ & Set of all intersections within the road network \\
$I$ & Set of population nodes \\
$P_{i j}$ & Set of (R-T)-Pareto-optimal paths between \\
& two population nodes $i$ and $j$ \\
$p$ & Path $\in P_{i j}$ consisting of arcs $(h, k)$ \\
$r_{h k}$ & Elementary catastrophe hazard of arc $(h, k)$ \\
$R_{p}$ & Risk of a path between two population nodes \\
\hline
\end{tabular}

considered for two approaches, assuming that whole areas located along a river are flooded: Minimal travel time and minimal risk.

Note that this paper does not focus on the determination of adequate risk values, but considers different approaches for measuring the risk of a distribution tour and the integration of these approaches in an optimization algorithm.

For the different risk approaches, it is necessary to determine the Pareto-optimal connections between each pair of population nodes (candidate locations for water tanks) regarding risk and travel time as objective functions to be minimized. These paths are called (R-T)-Pareto-optimal, where $R$ refers to risk and $T$ refers to travel time.

Figure 1 shows four (R-T)-Pareto-optimal connections, where the path on the left is the one with the smallest travel time but the highest risk value. Between the two population nodes, there exist further (R-T)-Pareto-optimal paths associated with larger travel times but lower risk values. The path on the right is the one with the largest travel time and the lowest risk value.

In Table 1, the notation for the different risk approaches is introduced.

The set of all intersections within the road network is denoted by $J$, while $I$ denotes the set of population nodes and candidate stopping points for the distribution of drinking water. Note that $I$ is a subset of $J(I \subseteq J)$. The risk between two population nodes is the probability that the connection between the two nodes does not remain accessible. The elementary catastrophe hazard $r_{h k}$ of each arc is based on catastrophe models for specific regions, which may be considered here as the probability of disruption on arc $(h, k)$. These values $r_{h k}$ are then used for the computation of the different risk approaches presented in the following.

For any (R-T)-Pareto-optimal path $p \in P_{i j}$ between two population centers, the total risk is determined by the maximal elementary catastrophe hazard of all arcs $(h, k)$ along that path, expressed by

$$
R_{p}=\max _{h k \in p}\left(r_{h k}\right)
$$


Considering the maximum is adequate, as the elementary catastrophe hazard values along a path are correlated and should therefore not be summed up. This is commonly done in hazardous material transportation (cf. Zografos and Androutsopoulos 2008).

Minimal travel time The minimal travel time approach considers the fastest out of all (R-T)-Pareto-optimal connections between each pair of population nodes, which is at the same time the connection with the maximal risk value, given by

$$
R_{i j}^{1}=\max _{p \in P_{i j}}\left(R_{p}\right)
$$

Minimal risk The minimal risk approach considers the safest out of all (R-T)-Paretooptimal connections between each pair of population nodes. Note that this connection is at the same time the one with the longest travel time, due to the concept of Pareto optimality. It is given as

$$
R_{i j}^{2}=\min _{p \in P_{i j}}\left(R_{p}\right)
$$

Number of alternative paths In contrast to the two approaches introduced so far, which consider the risk of a specific path to become impassable, the third approach takes the number of alternative (R-T)-Pareto-optimal paths between each pair of population nodes into consideration. This means that we do not measure the probability of a path to become impassable, but how many alternative (R-T)-Pareto-optimal connections could be used between two population centers. Paths between any two nodes are said to be alternative, if they differ at least in two arcs. We do not consider totally arc-disjoint paths, since, in a post-disaster situation in a developing country, it might be more appropriate to avoid critical arcs, such as bridges, that can cause the failure of a whole path even if all the other arcs remain accessible.

The number of alternative paths between two population nodes $i$ and $j$ is measured as

$$
R_{i j}^{3}=\left|P_{i j}\right|
$$

The values computed in this way are normalized to a scale from 0 to $1\left(R_{i j}^{3}\right.$ normalized $)$ and converted into a minimization formulation such that the lower the risk values, the more secure the path.

$$
R_{i j}^{3^{\prime}}=1-R_{i j}^{3 \text { normalized }}
$$

Unreachability We combine the characteristics of the risk approaches introduced so far into a global measure by considering not only the number of alternative paths between two population nodes but also their risk of becoming impassable. We call this approach unreachability, as it determines how vulnerable a population node elected as water distribution point is to be unreachable in the aftermath of a disaster. Unreachability is calculated as the sum of the risk values of all alternative (R-T)-Pareto-optimal 
connections between each pair of population nodes. In order to allow a useful combination of the number of paths and their associated risk values, the risk value of every alternative path between a pair of population nodes is first converted into the probability of remaining accessible. Thereafter these values are summed up.

$$
R_{i j}^{4}=\sum_{p \in P_{i j}}\left(1-R_{p}\right)
$$

$R_{i j}^{4}$ subsequently is normalized to a scale from 0 to $1\left(R_{i j}^{4}\right.$ normalized $)$, and converted into a minimization formulation.

$$
R_{i j}^{4^{\prime}}=1-R_{i j}^{4 \text { normalized }}
$$

Note that the more securely two adjacent population nodes can be reached, the larger the number of paths that connect the nodes and the lower the risk value(s) of the connection(s).

Threshold risk The threshold risk approach measures the risk of a path between two population nodes as the number of arcs belonging to a specific category of catastrophe hazard values larger than or equal to $\alpha$. Therefore, the number of arcs with an elementary catastrophe hazard equal to or larger than some value $\alpha$, belonging to each alternative ( $\mathrm{R}-\mathrm{T})$-Pareto-optimal connection between two population nodes, is determined, assuming independent catastrophe hazards of the arcs.

$$
R_{i j}^{5}=\max _{p \in P_{i j}}\left(R_{p}^{5}\right)
$$

where

$$
R_{p}^{5}=\left|\left\{h k \in p \mid \alpha \leq r_{h k} \leq 1\right\}\right|
$$

\subsubsection{Minisum facility location criterion and maximal covering location criterion}

Especially in a post-disaster situation, it is necessary to provide drinking water for population members within a distance that is reasonable for them to traverse. If the affected people have to cover a long distance in order to receive drinking water, they might prefer to use raw water from a source nearer to their homes, risking illnesses and epidemics. This is why we formulate the second objective as a combination of the minisum criterion and the MCLC. Both objective functions should be minimized and therefore also the formulation of the MCLC has to be converted into a minimization problem. For the computation of the objective functions to be practically feasible, we follow the usual approach in location analysis to define a set of population centers (nodes of a location graph), each representing a small geographical area by a single point.

The minisum objective function is computed by determining the shortest distance from each population center to the nearest stopping point. Each of these distance values is 
then multiplied by the number of inhabitants at the corresponding population center point and summed up over all population points.

The MCLC consists in minimizing the number of people that are not able to reach a water distribution point within a predefined reasonable walking distance, defined on the road network. The objective function value of the MCLC is the total number of inhabitants that cannot reach a water tank within this limit.

Note that each population center is assigned to its nearest water distribution point, even if it is not covered within the predefined reasonable walking distance.

The values of these two criteria are combined into one objective function with a weighted average. In our experiments, we chose the value 0.5 for each of these weights, assuming both objectives as equally important. Note that the minisum criterion represents a utilitarian point of view (maximum overall benefit). As the distances between a population center and the nearest water tank are weighted by the number of population members, people living in regions with fewer inhabitants are more likely to be confronted with long walking distances to the nearest water tank. Therefore, the MCLC objective considers equity, balancing the travel times of all population members.

\subsubsection{Minimization of tour length}

In a post-disaster situation, operations have to be carried out with a limited budget; therefore, tour lengths are to be minimized for economic reasons. After a natural catastrophe, roads might be difficult to traverse if they are damaged, which impacts the speed of relief vehicles. Therefore, it is more appropriate to calculate the costs in terms of travel time instead of distance. The total tour length is calculated by summing up the travel times of all arcs included in a tour.

\section{Problem description}

Table 2 introduces the notation for the CTP, which can be described as follows.

Let $G$ be a directed graph where $V$ is the vertex set and $A$ is the arc set. Vertices $v_{0}$ and $v_{n+1}$ represent the same depot, which is equipped with a limited amount of goods needed by the affected population. To be more specific, the depot contains a drinking water treatment plant and is located near an available raw water source. All tours start from vertex $v_{0}$, where $m$ vehicles of a certain capacity are placed, and end at vertex $v_{n+1}$. Zero demands are defined for these two nodes. Each vertex $v$ represents the population members living in the surrounding area that need to be served with drinking water. The arcs contained in $A$ represent the road network which is still available after a catastrophe. Via these arcs, $v_{1}$ to $v_{n}$ can be visited but no node has to be visited. A distance or travel time matrix satisfying the triangle inequality is defined on $A$.

Let $I$ denote the set of population nodes and candidate stopping points, where water is distributed. Let $K$ denote the set of homogeneous vehicles placed at the depot.

The data specifying the problem instance are the following: $w_{j}$ is the number of people in need at population node $j$, and $d_{i j}$ is the distance between vertices $i$ and $j$ $(i, j \in I)$. $Q$ denotes the maximum capacity of each vehicle. 
Table 2 Notation

\begin{tabular}{|c|c|}
\hline \multicolumn{2}{|c|}{ Constants } \\
\hline$V$ & Vertex set \\
\hline$A$ & Arc set \\
\hline$I$ & Set of population nodes \\
\hline$K$ & Set of homogeneous vehicles \\
\hline \multicolumn{2}{|l|}{ Data } \\
\hline$w_{j}$ & Number of people in need at population node $j$ \\
\hline$d_{i j}$ & Distance between vertices $i$ and $j$ \\
\hline$d_{\max }$ & MCLC distance threshold \\
\hline$Q$ & Maximum capacity of each vehicle \\
\hline$R_{i j}$ & Risk value of arc $(i, j)$ \\
\hline \multicolumn{2}{|c|}{ Variables } \\
\hline$z_{i k}$ & $\begin{array}{l}1 \text { if candidate location } i \text { is a stopping point in tour } \mathrm{k} \text {, } \\
0 \text { otherwise }\end{array}$ \\
\hline$x_{i j}^{k}$ & $\begin{array}{l}1 \text { if } \operatorname{arc}(i, j) \text { is included in the tour of vehicle } \mathrm{k} \text {, } \\
0 \text { otherwise }\end{array}$ \\
\hline$y_{j}$ & $\begin{array}{l}1 \text { if population node } j \text { has a stopping point within a distance } \leq d_{\max } \text {, } \\
0 \text { otherwise }\end{array}$ \\
\hline$u_{i j}$ & $\begin{array}{l}1 \text { if service for population node } j \text { is provided by the stopping point } i \text {, } \\
0 \text { otherwise }\end{array}$ \\
\hline$a_{i j}$ & $\begin{array}{l}1 \text { if } d_{i j} \leq d_{\max } \\
0 \text { otherwise }\end{array}$ \\
\hline$s_{i}$ & Number of capacity units required at candidate location $i$ \\
\hline$q_{i k}$ & Vehicle load after visiting stopping point $i$ \\
\hline
\end{tabular}

$R_{i j}(i, j \in I)$ are the risk values computed as outlined in Sect. 2.3.1. The binary variable $z_{i k}(i \in I, k \in K)$ is equal to 1 if candidate location $i$ is a stopping point in tour $k$, and 0 otherwise. $x_{i j}^{k}$ denotes the binary variable which is equal to 1 if arc $(i, j)$ is included in the tour of vehicle $k$, and 0 otherwise. The binary variable $y_{j}$ is equal to 1 if population node $j$ has a stopping point within a distance of less than or equal to $d_{\max }$, where the symbol $d_{\max }$ specifies the MCLC distance threshold, and 0 otherwise $(j \in I)$. The binary variable $u_{i j}$ is equal to 1 if service for population node $j$ is provided by the stopping point $i$, and 0 otherwise $(i, j \in I)$. The elements $a_{i j}$ are defined as 1 if $d_{i j}$ is less than or equal to $d_{\max }$, and 0 otherwise $(i, j \in I)$. The integer variable $s_{i}$ denotes the number of capacity units required at candidate location $i$. The integer variable $q_{i k}$ corresponds to the vehicle load after visiting stopping point $i$. Using this notation, the following multi-objective integer program can be written:

$$
\begin{gathered}
\left(f_{1}, f_{2}, f_{3}\right) \rightarrow \min \text { s.t. } \\
f_{1}^{\mathrm{MODL} 1}=\max _{i, j \in I, k \in K}\left(R_{i j}^{1} x_{i j}^{k}\right), \\
f_{2}=\gamma \sum_{j \in I} w_{j} \sum_{i \in I} d_{i j} u_{i j}+(1-\gamma) \sum_{j \in I} w_{j}\left(1-y_{j}\right),
\end{gathered}
$$




$$
\begin{aligned}
& f_{3}=\sum_{k \in K} \sum_{i, j \in I} d_{i j} x_{i j}^{k}, \\
& \sum_{i \in I} u_{i j}=1 \quad \forall j \in I \backslash\{0, n+1\}, \\
& \sum_{k \in K} z_{i k}-u_{i j} \geq 0 \quad \forall i, j \in I, \\
& \sum_{i \in I} \sum_{k \in K} a_{i j} z_{i k} \geq y_{j} \quad \forall j \in I, \\
& \sum_{j \in I} w_{j} u_{i j} \leq s_{i} \quad \forall i \in I, \\
& q_{i k}-s_{j}+\left(1-x_{i j}^{k}\right) M \geq q_{j k} \quad \forall i \in I \backslash\{n+1\}, \quad \forall j \in I \backslash\{0\}, \quad \forall k \in K, \\
& q_{i k} \leq Q \quad \forall i \in I, \quad \forall k \in K \text {, } \\
& \sum_{i \in I} d_{i j} u_{i j} \leq d_{m j}+M\left(1-\sum_{k \in K} z_{m k}\right) \quad \forall m, j \in I, \\
& \sum_{i \in I, i \neq p} x_{i p}^{k}-\sum_{j \in I, j \neq p} x_{p j}^{k}=0 \quad \forall p \in I \backslash\{0, n+1\}, \quad \forall k \in K, \\
& \sum_{i \in I} x_{i j}^{k} \leq 1 \quad \forall j \in I \backslash\{0, n+1\}, \quad \forall k \in K, \\
& \sum_{j \in I} x_{i j}^{k} \leq 1 \quad \forall i \in I \backslash\{0, n+1\}, \forall k \in K, \\
& \sum_{i \in I \backslash\{n+1\}} x_{i j}^{k}-z_{j k} \geq 0 \quad \forall j \in I, \forall k \in K, \\
& \sum_{k \in K} z_{i k} \leq 1 \quad \forall i \in I \backslash\{0, n+1\}, \\
& \sum_{j \in I \backslash\{0\}} x_{0 j}^{k}=1 \quad \forall k \in K, \\
& \sum_{j \in I \backslash\{n+1\}} x_{j, n+1}^{k}=1 \quad \forall k \in K, \\
& x_{i j}^{k} \in\{0,1\} \quad \forall i \in I \backslash\{n+1\}, \forall j \in I \backslash\{0\}, k \in K, \\
& u_{i j} \in\{0,1\} \quad \forall i, j \in I \text {, } \\
& z_{i k} \in\{0,1\} \quad \forall i \in I, k \in K \text {, } \\
& y_{j} \in\{0,1\} \quad \forall j \in I, \\
& q_{i k} \geq 0 \text { and integer } \forall i \in I, k \in K \text {, } \\
& s_{i} \geq 0 \text { and integer } \forall i \in I \text {. }
\end{aligned}
$$

Equation (11) represents the first objective function as the risk measured with the minimal travel time approach. The maximal risk value of all $\operatorname{arcs}(i, j)$ between two visited population centers is minimized. The multi-objective integer program is referred to as 
multi-objective disaster logistics problem for risk measure 1 (MODL 1). To adapt $f_{1}$ to each of the five risk measures, Eq. (11) is replaced by

$$
\begin{aligned}
f_{1}^{\mathrm{MODL} 2} & =\max _{i, j \in I, k \in K}\left(R_{i j}^{2} x_{i j}^{k}\right) \\
f_{1}^{\mathrm{MODL} 3} & =\max _{i, j \in I, k \in K}\left(R_{i j}^{3}{ }^{\prime} x_{i j}^{k}\right) \\
f_{1}^{\mathrm{MODL} 4} & =\max _{i, j \in I, k \in K}\left(R_{i j}^{4}{ }^{\prime} x_{i j}^{k}\right) \\
f_{1}^{\mathrm{MODL}} 5 & =\sum_{k \in K} \sum_{i, j \in I} R_{i j}^{5} x_{i j}^{k}
\end{aligned}
$$

Equation (34) represents the first objective function as the risk measured with the minimal risk approach. Equation (35) represents the first objective function as the risk measured with the number of alternative paths approach, while Eq. (36) represents the first objective function as the risk measured with the unreachability approach. For MODL 2 through MODL 4, the maximal risk value of all $\operatorname{arcs}(i, j)$ between two visited population centers is minimized. Equation (37) represents the first objective function as the risk measured with the threshold risk approach. For MODL 5, the risk values are summed up over all arcs $(i, j)$ included in the solution.

Equation (12) determines the second objective function as a weighted average of the minisum objective function and the MCLC objective function. Therein, $\gamma$ is a parameter with $0<\gamma<1$. Equation (13) represents the overall tour length. Constraints (14) require that each population node has a stopping point assigned. Constraints (15) ensure that only a candidate location where a stopping point has been planned can provide service, and constraints (16) ensure that population node $j$ can only be serviced within distance $d_{\max }$ if there is a node $i$ within distance $d_{\text {max }}$ where water is available. Constraints (17) guarantee that the demand occurring at stopping point $i$ is covered by the capacity units at this location. Constraints (18) force the continuous decrease of the load of each vehicle. Through these constraints, subtours are eliminated as well. Constraints (19) are capacity restrictions. Constraints (20), where $M$ denotes a very large number, express the assumption that inhabitants at a certain population node $j$ always choose the stopping point that is nearest to node $j$ : Observe that the left-hand side of (20) gives the distance from population node $j$ to the stopping point to which $j$ is assigned, and the right-hand side gives the distance from population node $j$ to any (other) point $m$, if $m$ is a stopping point, and a very high value otherwise. Constraints (21)-(23) are degree constraints, regulating the number of arcs going in and out of a population center. Constraints (24) require that at a candidate location, a stopping point can only be planned when an arc that leads to this point is included in the tour. Constraints (25) state that each stopping point can only be visited by one vehicle. Constraints (26) and (27) force each vehicle to leave and enter the depot. The remaining constraints restrict the variables to binary or integer values. Note that variables $q_{i k}$ and $s_{i}$ will automatically be integer; therefore, they could also be declared as continuous without changing the optimal solution. 


\section{Solution procedure}

An adapted version of a multi-objective MA is applied to the minimal risk approach, the number of alternative paths approach and the unreachability approach. For the minimal travel time approach and the threshold risk approach, the MA is extended by an enrichment phase, that contributes to obtaining good compromise solutions for the potentially Pareto-optimal frontier. Therefore, the minisum criterion and MCLC value of each solution is fixed after executing the MA, while travel time and risk are investigated in detail. The procedure, illustrated in Fig. 2, allows an enrichment of the potentially Pareto-optimal frontier.

The whole process performs as follows: In phase 1, a potentially Pareto-optimal frontier of the three-objective (risk, minisum \& MCLC, travel time) problem is determined by our MA. Location decisions for water tanks (population nodes visited within the CTP) and, as a consequence, the objective function value for minisum criterion and MCLC are fixed. In phase 2, by applying Martins' algorithm, paths between two nodes in a solution can be modified. Therefore, all possible combinations of paths between the nodes included in the potentially Pareto-optimal solutions are computed. Between each pair of nodes, every alternative path is combined with each alternative path between all the other nodes. The solutions found in this way are evaluated considering only travel time and risk as objectives (the objective function value for minisum criterion and MCLC has been fixed before) and dominated ones are eliminated. The remaining bi-objective (R-T)-Pareto-optimal solutions are regarded in detail in order to update the travel time and risk matrices used for the MA. This means that, for each of the connections between two nodes contained in the remaining Pareto set approximation, the travel time and risk values are transferred to the matrices used in the three-objective MA. The whole process is repeated until the received potentially Pareto-optimal frontier cannot be improved. With this procedure, it is possible to generate potentially Pareto-optimal solutions with lower risk values that would not have been discovered otherwise. This is due to the fact that the original matrix only contains the shortest paths and their associated risk values. By applying the enrichment phase, it is possible to find paths with potentially longer travel times but smaller risk values.

The enrichment phase is performed for the minimal travel time approach and the threshold risk approach. For the other approaches, it is not useful, as for minimal risk this could only generate higher risk values. Number of alternative paths and unreachability are global measures already considering all existing travel time and risk values between two nodes, where this procedure cannot be applied.

\subsection{Phase 1: Memetic algorithm}

We developed a memetic solution approach in order to generate a set of potentially Pareto-optimal solutions for our multi-objective CTP. The method is based on the Nondominated Sorting Genetic Algorithm II (NSGA-II) by Deb et al. (2002), including a variable neighborhood search (VNS) (cf. Hansen and Mladenović 1997; Hansen andMladenović 2003) as well as path relinking (cf. Glover and Laguna 1997). Like all genetic algorithms, NSGA-II is an iterative, population-based search procedure. 
Fig. 2 Solution procedure

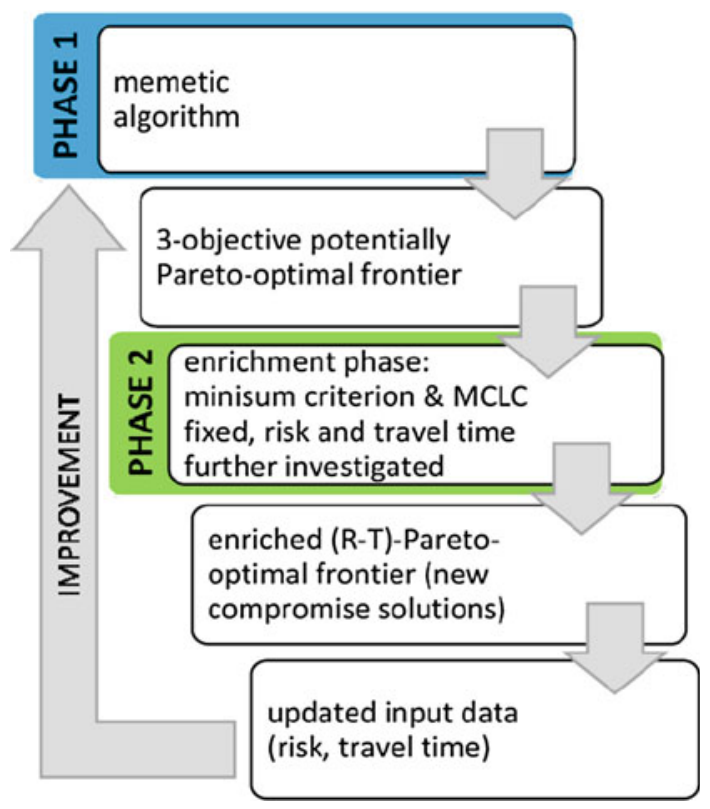

NSGA-II computes a series of so-called generations, where each generation consists of a population of feasible solutions of the optimization problem under consideration. Diversification and intensification strategies are applied to each generation. We perform a VNS where NSGA-II originally proposes genetic operators in order to create a new generation. NSGA-II provides a good basis for location decisions, which in our problem concern the stops of the vehicles along their tours (positions for water tanks). For the routing part itself, VNS is preferred, complemented with path relinking, as the classical genetic operators originally included in the NSGA-II pose possible difficulties concerning the generation of feasible solutions.

In our algorithm, $M$ solutions (chromosomes) are chosen out of the current population that will undergo a VNS, preferring solutions that belong to a fitter frontier in the objective space. This means that solutions which are fitter (better with respect to the objective function values) have a higher probability of being selected. Now, interroute move and swap operations are applied to the chosen chromosomes, aiming at improving solution quality. The move operator inserts a tour stop (gene) of one partial tour into another partial tour. It is also possible to include a tour stop that belongs to the set of nodes that are not visited in the current solution or exclude a tour stop from the solution. The swap operator exchanges tour stops between different partial tours. Again, a tour stop could also be swapped with a node currently not included in the solution.

We do not use recombination as in the classical NSGA-II. Therefore, in order to combine characteristics of good solutions, path relinking is applied to the nondominated solutions created with the procedure described above. Path relinking is a heuristic, intensifying the search procedure by generating paths between elite solutions, originally developed by Glover and Laguna (1997). In each iteration, the initial and guiding 


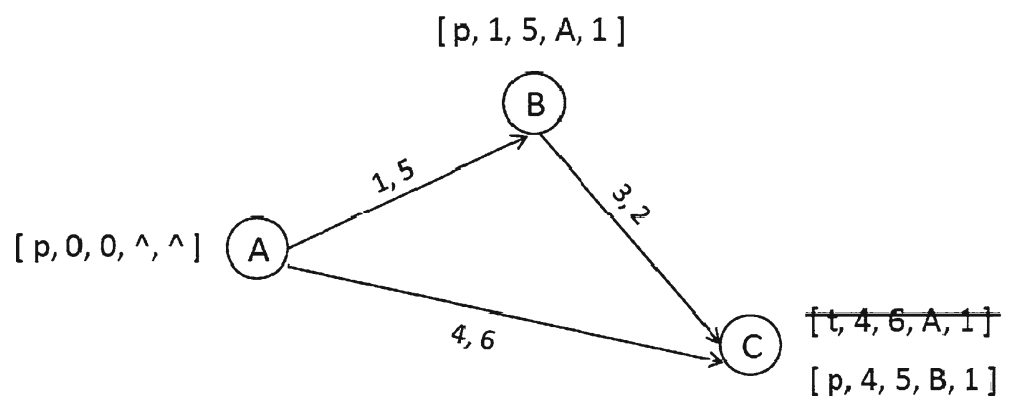

Fig. 3 Example of Martins' algorithm for three nodes. Two paths lead to node C, each represented by a label. Path $\mathrm{A} \rightarrow \mathrm{C}$ is dominated and therefore the first label at node $\mathrm{C}$ is crossed out. The remaining label represents a Pareto-optimal path $\mathrm{A} \rightarrow \mathrm{B} \rightarrow \mathrm{C}$

solutions are randomly chosen from the current best frontier, regarding each solution only once. A shortest path between each pair of solutions is generated by a decreasing edit distance (cf. Sörensen 2007). The edit distance between two solution representations is the minimum number of edit operations required to transform the initial solution into the guiding solution. Edit operations could be reversals, insertions, or deletions of tour stops (population centers, where water tanks are placed). Experimental results showed that the number of Pareto-optimal solutions found by the memetic algorithm is improved by $40 \%$ when path relinking is applied.

\subsection{Phase 2: Enrichment}

Martins' algorithm for multi-objective shortest paths (cf. Gandibleux et al. 2006) is applied to generate good compromise solutions within the enrichment phase. The Martins' algorithm is a multiple objective version of the Dijkstra algorithm. Instead of a 'min' operator, a dominance test is used in order to determine the Pareto-optimal shortest paths. Starting from the source node, each node receives a temporary label where, in each iteration, the lexicographically smallest label of a neighbor is converted into a permanent one and its information is propagated to all the temporary labels of its successors. A label contains information on the objective function values of a specific path. This means that each label consists of five entries, namely the state of the label (permanent or temporary), objective function value travel time, objective function value risk, identity of its predecessor and position of the related label at the predecessor vertex. In each iteration, the temporary label of a vertex with the smallest travel time and, among those with the same travel time, the label with the smallest risk value is made permanent. For all nodes adjacent to this vertex, the newly accessible paths are created. At each node, a dominance test considering travel time and risk is performed, comparing the new path to the already existing labels. If the new path is not dominated, it is stored as a temporary label. The whole procedure stops when all dominated labels have been deleted and all remaining temporary labels have been converted into permanent labels, each representing a Pareto-optimal path. An example is presented in Fig. 3.

As objective function values for the paths between two nodes costs and risk are considered. The costs are measured in terms of travel times, which are summed up 
over all arcs. The risk of a given path is determined according to the risk approaches presented in Sect. 2.3.1. Note that risk is modeled as a bottleneck objective function. Both objective function values are minimized. The Pareto set approximation computed within the MA is extended in the enrichment phase. The population center nodes to be visited are kept as determined within the MA. For each solution with Martins' algorithm, the (R-T)-Pareto-optimal connections between consecutive nodes in a tour are computed. Within a solution, all combinations of connections between the included nodes are considered and only non-dominated ones are stored. The (R-T)Pareto frontier created in this way is used for updating the input data in order to create additional potentially Pareto-optimal solutions. Between each pair of nodes, the travel time and risk values are compared with the matrices used for the MA. If the new risk value between a pair of nodes is smaller than the one used before, the travel time and risk matrices are updated for the execution of the MA and the whole procedure is repeated. This solution method allows the Pareto set approximation to be enriched by introducing risk values that are not available within the original risk matrix.

\section{Computational experiments}

We tested the proposed solution approach on two real-world instances from Manabí, Ecuador. We applied the five risk measures introduced in Sect. 2.3.1 and evaluated them by comparing each risk measure to the usual approach of considering shortest paths, here called minimal travel time. It is shown that the approximated Pareto set can be enriched by our two-phase approach.

\subsection{Test instances}

The Manabí province is situated in north-western Ecuador, adjacent to the Pacific Ocean. Manabí is predominantly characterized by agricultural activities, being the most important coffee-growing region in the country. The province, which covers an area of 18,400 square kilometers, is exposed to the risk of earthquakes due to its location at the subduction zone of the Nazca Plate under the South American Plate. The risk values used for our experiments are based on forecasts of aftershocks following an earthquake. The rate of aftershocks of a specific magnitude can be calculated based on a stochastic model by Reasenberg and Jones (1994), depending on the distance to the mainshock and the mainshock magnitude.

We divided the whole province into a southern part and a northern part, allowing us to evaluate our risk approaches on the basis of two different real-world instances. It is assumed that an earthquake with a magnitude of 6.5 on the Richter scale occurred in Manabí. For each instance, one drinking water treatment plant is located at the depot, where a certain number of trucks are placed. Population centers represent the inhabitants living in the surrounding area that need to be supplied with drinking water. The maximum travel time along the available roads from any population point to the nearest tour stop where drinking water is provided is assumed as two hours. Any population point that lies within that travel time from a tour stop is considered as covered. 
Table 3 Example of the performance measure

\begin{tabular}{lll}
\hline & $\mathrm{A}$ & $\mathrm{B}$ \\
\hline $\mathrm{A}$ & - & 0.2 \\
$\mathrm{~B}$ & 0.6 & - \\
\hline
\end{tabular}

Aspiration levels for the different objective function values were introduced in order to avoid solutions, where none of the vehicles leaves the depot.

For our investigation, 12 potential locations for water tanks were determined for each instance as input data for the MA. With Martins' algorithm, 79 intersections of the road network were regarded for Manabí North, while Manabí South consists of 138 intersections.

\subsection{Performance measure}

In order to compare the solutions of two Pareto set approximations regarding their multi-objective nature, we applied the following performance measure:

Performance measure (PM): For a mutual comparison of the sets $A$ and $B$ produced by two heuristic algorithms, we applied a performance measure introduced by Zitzler and Thiele (1999).

$$
P M(A, B)=\frac{\left|\left\{z^{2} \in B \mid \exists z^{1} \in A: z^{1} \succeq z^{2}\right\}\right|}{|B|},
$$

where $z^{1} \succeq z^{2}$ if $z^{1}$ dominates $z^{2}$ or $z^{1}=z^{2}$.

The higher $P M(A, B)$ compared with $P M(B, A)$, the better is $A$ compared with $B$. For a better understanding of the performance measure, an example is presented in Table 3. It is shown that $60 \%$ of the solutions in set $A$ are (weakly) dominated by set $B$, while only $20 \%$ of the latter are dominated by or equal to the solutions in $A$.

\subsection{Numerical results}

For the real-world instances of Manabí North and Manabí South, sets of potentially Pareto-optimal solutions have been identified by our (two-phase) solution approach within a few minutes. The computing capacity required for running the algorithm is provided by a state-of-the-art personal computer, which makes the proposed solution approach suitable for humanitarian operations.

In order to give an overview of the alternative risk measures, Table 4 presents the range of the risk values as well as the associated travel time range. The values were computed by the MA for each of the respective risk measures $R_{i j}^{1}$ through $R_{i j}^{5}$, using MODL 1 through MODL 5, without an enrichment phase. Six runs with different random numbers were performed for each test instance. For making the values comparable, the risk values that correspond to MODL 2, 3, and 4 were evaluated with risk of minimal travel time and dominated solutions were eliminated. The minimal travel 
Table 4 Range of travel time and risk values without enrichment phase

\begin{tabular}{|c|c|c|c|c|c|c|}
\hline & \multicolumn{2}{|c|}{ Min travel time } & \multicolumn{2}{|l|}{ Min risk } & & \\
\hline & Travel time & $\operatorname{Risk}\left(R_{i j}^{1}\right)$ & Travel time & $\operatorname{Risk}\left(R_{i j}^{1}\right)$ & & \\
\hline Manabí North & $0.19-0.34$ & $0.95-0.99$ & $0.55-0.6$ & 0.9 & & \\
\hline \multirow[t]{3}{*}{ Manabí South } & $0.25-0.61$ & $0.97-0.98$ & $0.58-0.98$ & $0.81-0.83$ & & \\
\hline & \multicolumn{2}{|c|}{ Number of paths } & \multicolumn{2}{|c|}{ Unreachability } & \multicolumn{2}{|l|}{ Risk $>0.95$} \\
\hline & Travel time & $\operatorname{Risk}\left(R_{i j}^{1}\right)$ & Travel time & $\operatorname{Risk}\left(R_{i j}^{1}\right)$ & Travel time & $\operatorname{Risk}\left(R_{i j}^{1}\right)$ \\
\hline Manabí North & $0.19-0.41$ & $0.95-0.99$ & $0.19-0.4$ & $0.95-0.99$ & $0.19-0.36$ & $0.95-0.99$ \\
\hline Manabí South & $0.25-0.56$ & $0.97-0.98$ & $0.25-0.59$ & $0.97-0.98$ & $0.26-0.68$ & $0.97-0.98$ \\
\hline
\end{tabular}

Table 5 Range of travel time and risk values with enrichment phase for risk of minimal travel time

\begin{tabular}{llllll}
\hline & \multicolumn{2}{l}{ Before enrichment } & & & \multicolumn{2}{l}{ After enrichment } \\
\cline { 2 - 3 } \cline { 5 - 6 } & Travel time & Risk & & Travel time & Risk \\
\hline Manabí North & $0.19-0.34$ & $0.95-0.99$ & & $0.19-0.55$ & $0.9-0.99$ \\
Manabí South & $0.25-0.61$ & $0.97-0.98$ & & $0.25-0.87$ & $0.81-0.98$ \\
\hline
\end{tabular}

time approach and the minimal risk approach can be compared based on their original values. A risk value of 0.99 indicates that the given solution has a probability of $99 \%$ of becoming impassable in case of an aftershock.

Considering the minimal travel time approach, which takes the maximal risk values into account, we were able to enrich the potential Pareto frontier, as presented in Table 5. For Manabí North, the risk values initially range from 0.95 to 0.99 . By updating the travel time and risk matrices, the risk values of the solutions proposed by the MA can be enriched, now ranging from 0.9 to 0.99 . For Manabí South, the results are even more significant, as presented in Fig. 4. Note that, by updating the travel time and risk matrices, the solutions first shift to the right due to the longer travel times (from the triangles to the dots). In the same iteration, however, new solutions are determined containing lower risk values. With each additional iteration, travel times decrease, maintaining the lower risk values, represented by circles. It can easily be observed that the solutions of the initial (R-T)-Pareto frontier are reached again at the third iteration, but enriched by a new solution which is better with respect to risk (denoted by the circle). The risk values of the solutions contained in the initial potentially Pareto-optimal frontier range from 0.97 to 0.98 . By updating the travel time and risk matrices, the risk values of the solutions proposed by the MA can be enriched, now ranging from 0.81 to 0.98 .

The minimal risk approach measures the probability of a path to become impassable by assessing the minimal risk value of a connection between two population nodes and the associated travel time. 


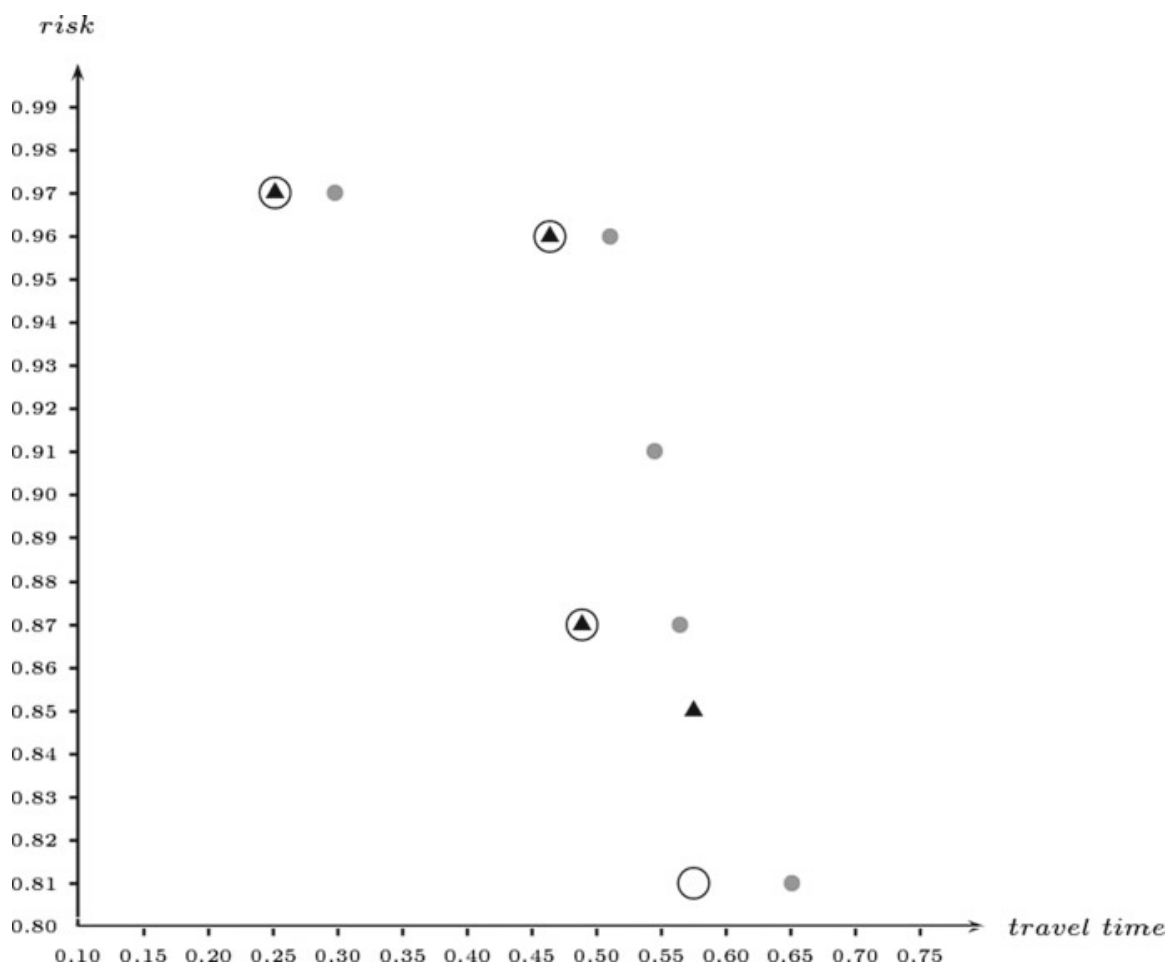

Fig. 4 Enrichment process. Triangles represent the (R-T)-Pareto-optimal solutions found in the first iteration of the enrichment procedure, dots represent the (R-T)-Pareto-optimal solutions of the second iteration, and circles represent the (R-T)-Pareto-optimal solutions determined in the last iteration of the enrichment procedure

Regarding the whole set of potentially Pareto-optimal solutions proposed by our three-objective MA, the risk values in general are significantly lower using the minimal risk approach compared with the set of solutions achieved using the minimal travel time approach. For Manabí North, the potentially Pareto-optimal frontier for minimal risk contains only paths with a probability of 0.9 to become impassable (travel time 0.55-0.6), while, for minimal travel time, they range from 0.95 to 0.99 (travel time 0.19-0.34). For Manabí South, the potentially Pareto-optimal frontier for minimal risk contains only paths with a probability of 0.81 to 0.83 to become impassable (travel time $0.58-0.98$ ). The risk values in the set of potentially Pareto-optimal solutions considering minimal travel time range from 0.97 to 0.98 (travel time $0.25-0.61$ ).

The values of $P M$ presented in Table 6 indicate that the two sets of potentially Pareto-optimal solutions are equally good regarding all objective functions simultaneously. This means that where the minimal travel time approach is good with respect to travel time, the minimal risk approach performs worse, while the opposite holds for the risk values. Therefore, for both risk measures, the $P M$ values are 0.0 , expressing that all potentially Pareto-optimal solutions generated regarding the minimal travel time approach are better than the solutions belonging to the minimal risk approach with respect to travel time, and vice versa with respect to risk. 
Table $6 P M$ values for minimal risk

\begin{tabular}{llllll}
\hline & \multicolumn{2}{l}{ Manabí North } & & \multicolumn{2}{l}{ Manabí South } \\
\cline { 2 - 3 } \cline { 5 - 6 } & Min travel time & Min risk & & Min travel time & Min risk \\
\hline Min travel time & - & 0.0 & - & 0.0 \\
Min risk & 0.0 & - & 0.0 & - \\
\hline
\end{tabular}

Table $7 \quad P M$ values for number of alternative paths

\begin{tabular}{llllll}
\hline & \multicolumn{2}{l}{ Manabí North } & & \multicolumn{2}{l}{ Manabí South } \\
\cline { 2 - 3 } \cline { 5 - 6 } & Min travel time & Number of paths & & Min travel time & Number of paths \\
\hline Min travel time & - & 0.9189 & - & 0.4615 \\
Number of paths & 0.5918 & - & 0.3333 & - \\
\hline
\end{tabular}

As an alternative to the first two approaches, which take correlated risk values into consideration, we developed an approach that counts the number of alternative (R-T)-Pareto-optimal paths between two nodes. In this way, it is determined how many alternative paths could be used in case of failure of one route, assuming independent risk values. However, this approach is weak in comparison with the other ones, as it just counts all (R-T)-Pareto-optimal connections between two population nodes that contain at least two disjoint arcs. This means that, possibly, all alternative connections could become impassable at once if, for instance, a bridge that they all have in common is destroyed by an aftershock.

Table 7 presents the values of performance measure $P M$ for the Pareto set approximation computed with the minimal travel time approach as the first objective or the number of alternative paths approach, respectively, both evaluated with risk of minimal travel time. Therefore, the risk values that correspond to the number of alternative paths approach are evaluated with minimal travel time and dominated solutions are eliminated.

Note that the set of solutions generated with the minimal travel time approach and the associated risk values outperform the Pareto set approximation generated with the number of alternative paths approach. While for Manabí South, for example, $33 \%$ of solutions considering minimal travel time are dominated by or equal to the solutions considering number of alternative paths, $46 \%$ of the latter are at least weakly dominated.

In order to cope with the shortcomings of the number of alternative paths approach, another risk measure was developed, called unreachability. It represents a global measure that takes the number of (R-T)-Pareto-optimal paths between two nodes as well as their particular risk values into consideration. Unreachability is calculated as the sum of the risk values of all alternative (R-T)-Pareto-optimal connections between each pair of population nodes.

Table 8 shows that the unreachability approach generates a set of solutions that is more secure measured in terms of risk associated with minimum travel time, than the minimal travel time approach. Performance measure PM expresses this relationship, 
Table $8 P M$ values for unreachability

\begin{tabular}{llllll}
\hline & \multicolumn{2}{l}{ Manabí North } & & & Manabí South \\
\cline { 5 - 6 } & Min travel time & Unreachability & & Min travel time & Unreachability \\
\hline $\begin{array}{l}\text { Min travel } \\
\text { time }\end{array}$ & - & 0.9111 & - & 0.4074 \\
Unreachability & 0.5714 & - & & 0.6111 & - \\
\hline
\end{tabular}

Table $9 P M$ values for threshold risk

\begin{tabular}{|c|c|c|c|c|}
\hline & \multicolumn{2}{|c|}{ Manabí North } & \multicolumn{2}{|l|}{ Manabí South } \\
\hline & $\begin{array}{l}\text { Min travel } \\
\text { time }\end{array}$ & Risk $>0.95$ & Min travel time & Risk $>0.95$ \\
\hline Min travel time & - & 0.975 & - & 0.4444 \\
\hline Risk $>0.95$ & 0.4694 & - & 0.3889 & - \\
\hline
\end{tabular}

where about $60 \%$ of solutions considering minimal travel times and the associated risk values are (weakly) dominated, while only $40 \%$ of the Pareto set approximation generated with the unreachability approach are dominated by or equal to these solutions.

For Manabí North, there is no significant dominance of the unreachability approach, which is due to the fact that this problem instance is limited by certain risk values. The road network in developing countries is sparse; therefore, certain arcs are included in almost all paths, as without them no connection would be possible. Given that we minimize the maximum of minimal risk values, specifically for the small instance Manabí North, the risk values are not quite diverse. Therefore, with the three-objective MA, the dominance is basically determined by the minisum criterion and MCLC and the travel time. However, in the real-world context, the area under investigation is larger, including diverse risk values, which allows the computation of a secure Pareto set approximation with the unreachability approach, as shown with Manabí South.

The threshold risk approach considers the number of arcs belonging to a connection between two population centers that have a catastrophe hazard value larger than 0.95 . Table 9 shows that threshold risk is outperformed by the minimal travel time approach.

By investigating travel time and risk in the enrichment phase for the threshold risk approach, its Pareto set approximation can be improved. Figure 5 provides a comparison of the bi-objective Pareto frontiers of minimal travel time and threshold risk for Manabí South. Therefore, the best improved (R-T)-Pareto-optimal frontiers of both measures are considered, evaluated with risk of minimal travel time. It can be observed that the solutions generated with threshold risk (dots) are dominated by the solutions generated with the risk values that correspond to minimal travel time (circles). For a better understanding of the two approaches, detailed representations of two solutions highlighted by an ellipse are presented in Figs. 6 and 7. Both solutions share almost the same objective function values with respect to minisum criterion and MCLC as well as travel time; they only differ in their risk values. The maps show Manabí South, where the black square is the depot, that contains a drinking water treatment plant and a given number of trucks. The points are the population centers, 


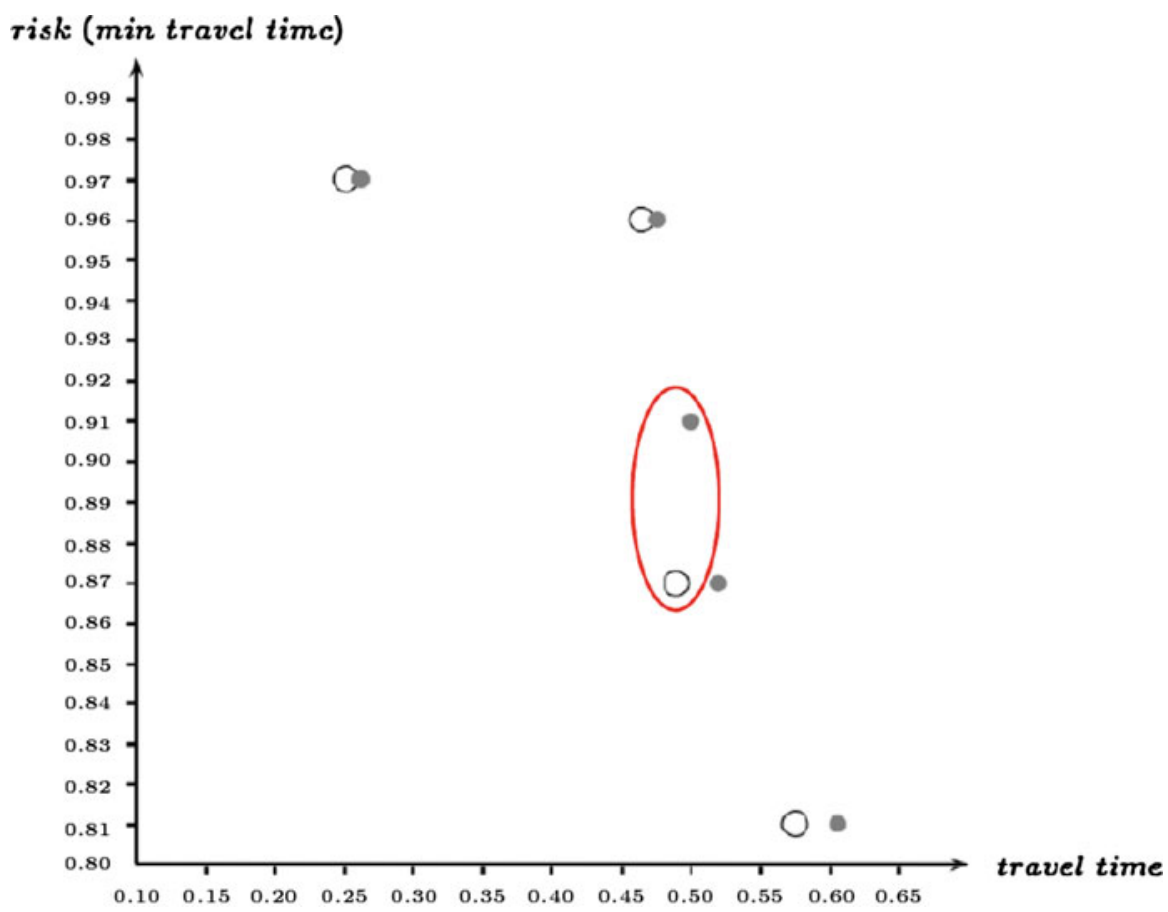

Fig. 5 Minimal travel time (circles) versus threshold risk (dots), evaluated with risk of minimal travel time

Fig. 6 Minimal travel time (risk 0.87, travel time 0.49)

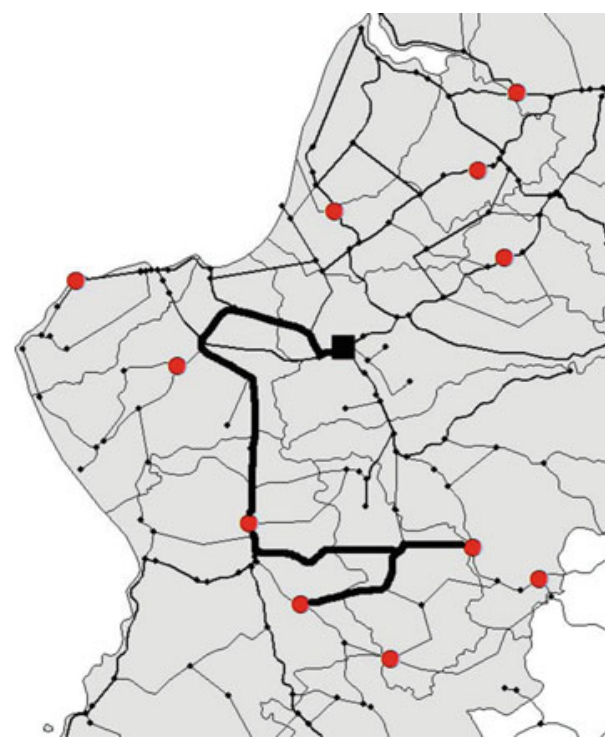

that need to be supplied with water. Trucks transport drinking water to population center points, where water tanks with tap stands are installed, such that all population members can get to their nearest distribution point for drinking water. While the solu- 
Fig. 7 Threshold risk (risk 0.91, travel time 0.5)

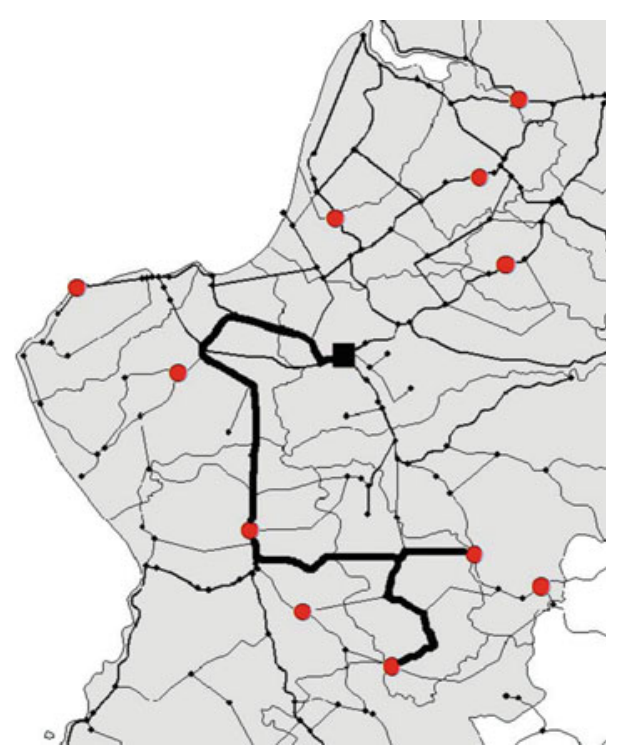

tion generated with the minimal travel time approach, presented in Fig. 6, has a risk of $87 \%$ of becoming impassable, the solution in Fig. 7, calculated with threshold risk, is threatened by a $91 \%$ failure.

The weakness of the threshold risk approach is its inability to consider the specific risk values of arcs that are below $\alpha$. This means that the threshold risk approach, in contrast to the minimal travel time approach, does not distinguish between the different risk values below the category of 0.95 and as a consequence fails to minimize risk values below $\alpha$.

To conclude, it can be observed that the unreachability approach provides the most global and efficient approach for measuring the risk on the paths of the water distribution tours. It is also shown that by applying the proposed enrichment procedure, (potentially) Pareto-optimal solutions can be determined that would not be found otherwise. The runtime of the proposed solution method requires a few seconds for the MA and minutes for the enrichment phase. The heuristic nature of the algorithm allows the procedure to be stopped at any time while still providing feasible solutions.

\section{Conclusion and implications}

In this paper, we presented a two-phase solution approach to solve a real-world motivated disaster relief problem for the delivery of drinking water in a post-disaster situation. We applied and analyzed five approaches for measuring the risk of the distribution tours to become impassable. The post-disaster situation under consideration is characterized by a high level of uncertainty. For example, an earthquake could by accompanied by aftershocks, floods by a rising water level over time, making delivery tours impassable. Robust tours for the distribution of drinking water are desirable for several reasons. First of all, it is important for the affected population to be able to 
rely on relief supplies on a regular basis at certain locations. Furthermore, for disaster relief organizations, changing daily tours requires significant organizational and administrative efforts.

Therefore, risk is considered as an objective function in our problem formulation and measured with one of the five alternative risk approaches presented in this paper. Experimental results showed that the unreachability approach is most suitable for measuring the risk of a delivery tour to become impassable in the aftermath of a disaster. Unreachability is calculated as the sum of the risk values of all alternative (R-T)-Pareto-optimal connections between a pair of population nodes. Our two-phase solution approach allows potentially Pareto-optimal solutions to be identified that cannot be found otherwise. By considering distribution tours with possibly longer travel times, the associated risk values can be reduced. Furthermore, by applying the twophase approach, it is possible to identify feasible solutions for each intended level of risk. In general, the multi-objective solution approach presented in this paper provides decision makers with a diverse set of feasible solutions, possessing different qualities with respect to the conflicting objectives. It is worth mentioning that the conflicting objectives cannot be optimized simultaneously. Therefore, there is a trade-off between short distribution tours, where population members have to travel a longer distance to reach the nearest water distribution point, and comparatively long routes but good coverage. It is crucial to determine a set of potentially Pareto-optimal solutions. This enables decision makers to choose a solution of this diverse set, according to their preferences for the different objectives (e.g. risk, coverage, travel time), to optimally provide the affected population with the available resources.

To make our approach practically useful and relevant, the described solution method has to be included into a decision support system that helps to find appropriate measures for disaster relief. Therefore information on topography and population is needed, which is available as electronic data. To be more specific, distances of the road network still available after the disaster are required, which are converted into travel times for the relief vehicles. Furthermore, the number of inhabitants in the affected area has to be known in order to specify demand points (population centers). The risk values are based on forecasts of aftershocks following an earthquake, which depend on the distance to the mainshock and the mainshock magnitude. The electronic data are then included into our algorithm, which provides the decision makers with a set of potentially Pareto-optimal solutions. In this way, the decision makers can consider the different solutions visualized on electronic maps of the affected region. Data generation in the aftermath of a disaster represents an upcoming discipline. The applicability of our solution approach is limited by the required data. However, current research in this discipline ameliorates the availability of relevant information for disaster relief operations. Geodata can be assessed via satellite images and information on topography and the extent of destruction can be measured via laser scanners. Currently, relevant information has to be partly assessed manually in case of an emergency. However, research is being undertaken aiming at extracting data out of satellite images automatically (cf. Lang et al. 2010; Tiede et al. 2010). Satellite and airborne imagery are rich sources of information about the real world, which allow the integration of imagebased information and existing geodata in the context of 'rapid mapping', the quick provision of maps for crisis management. 
Further application areas for the multi-objective covering tour model beyond disaster relief could be found in the context of development aid or refugee camp management. Clearly, the proposed model does not integrate all aspects of different disaster situations. Therefore, future research could concentrate on various mitigation activities that could restore infrastructure more quickly. An evaluation of these activities, such as land use controls to prevent occupation of high hazard areas or barrier construction to deflect disaster forces (cf. Altay and Green 2006), in the face of post-disaster conditions could be addressed in the future.

As we must face the possibility that, because of climate change effects, millions of people living in developing countries could be threatened by floods and related disasters within the next decades, models and solution techniques quite similar to those presented in this paper should be highly desirable.

Acknowledgments We thank the anonymous reviewers for their valuable and very useful comments. We also thank Stéphane Dauzère-Pérès for his careful proofreading. Financial support from the Austrian Science Fund (FWF) by grant \#L362-N15 is gratefully acknowledged. Moreover, the present research work has been partly supported by the International Campus on Safety and Intermodality in Transportation, the Nord-Pas-de-Calais Region, the European Community, the Regional Delegation for Research and Technology, the Ministry of Higher Education and Research, and the National Center for Scientific Research. The authors gratefully acknowledge the support of these institutions.

Open Access This article is distributed under the terms of the Creative Commons Attribution Noncommercial License which permits any noncommercial use, distribution, and reproduction in any medium, provided the original author(s) and source are credited.

\section{References}

Altay N, Green WG (2006) OR/MS research in disaster operations management. Eur J Oper Res 175(1):475-493

Balcik B, Beamon B, Smilowitz K (2008) Last mile distribution in humanitarian relief. J Intell Transp Syst 12:51-63

Barbarosoglu G, Özdamar L, Cevik A (2002) An interactive approach for hierarchical analysis of helicopter logistics in disaster relief operations. Eur J Oper Res 140:118-133

Campbell AM, Vandenbussche D, Hermann W (2008) Routing for relief efforts. Transp Sci 42(2):127-145

Clímaco JCN, Pascoal MMB (2009) Finding non-dominated bicriteria shortest pairs of disjoint simple paths. Comput Oper Res 39(11):2892-2898

De Angelis V, Mecoli M, Nikoi C, Storchi G (2007) Multiperiod integrated routing and scheduling of World Food Programme cargo planes in Angola. Comput Oper Res 34:1601-1615

Deb K, Pratap A, Agarwal S, Meyarivan T (2002) A fast and elitist multiobjective genetic algorithm: NSGA-II. IEEE Trans Evol Comput 6(2):182-197

Doerner K, Focke A, Gutjahr W (2007) Multicriteria tour planning for mobile healthcare facilities in a developing country. Eur J Oper Res 179:1078-1096

Fortz B (2000) Design of survivable networks with bounded rings. Kluwer Academic Publishers, Dordrecht

Gandibleux X, Beugnies F, Randriamasy S (2006) Martins' algorithm revisited for multi-objective shortest path problems with a MaxMin cost function 4OR 4:47-59

Gendreau M, Laporte G, Semet F (1997) The covering tour problem. Oper Res 45(4):568-576

Glover F, Laguna M (1997) Tabu Search. Kluwer Academic Publishers, Dordrecht 111

Gouveia L, Patrício P, de Sousa A (2008) Hop-constrained node survivable network design: an application to MPLS over WDM. Netw Spatial Econ 8(1):3-21

Hansen P, Mladenović N. (1997) Variable neighborhood search. Comput Oper Res 24:1097-1100

Hansen P, Mladenović N (2003) Variable neighbourhood search. In: Glover F, Kochenberger GA (eds) Handbook of metaheuristics, pp 145-158 
Hodgson MJ, Laporte G, Semet F (1998) A covering tour model for planning mobile health care facilities in Suhum district, Ghana. J Reg Sci 38(4):621-639

Kovács G, Spens KM (2007) Humanitarian logistics in disaster relief operations. Int J Phys Distrib Logist Manag 37(2):99-114

Kovács G, Tatham P (2009) Responding to disruptions in the supply network-from dormant to action. J Bus Logist 30(2):215-219

Lang S, Tiede D, Holbling D, Fureder P, Zeil P (2010) Earth observation (EO)-based ex post assessment of internally displaced person (IDP) camp evolution and population dynamics in Zam Zam, Darfur. Int J Remote Sens 31(21):5709-5731

Matisziw TC, Murray AT (2009) Modeling s-t path availability to support disaster vulnerability assessment of network infrastructure. Comput Oper Res 36(1):16-26

McLachlin R, Larson P, Khan S (2009) Not-for-profit supply chains in interrupted environments: the case of a faith-based humanitarian organisation. Manag Res News 32(11):1050-1064

Nolz P, Doerner K, Gutjahr W, Hartl R (2009) A Bi-objective metaheuristic for disaster relief operation planning. In: Coello CA, Dhaenens C, Jourdan L (eds) Advances in multi-objective nature inspired computing. Springer, Berlin, pp 169-191

Özdamar L, Ediz E, Beste K (2004) Emergency logistics planning in natural disasters. Ann Oper Res 129:217-245

Peduzzi P, Dao H, Herold C, Mouton F (2009) Assessing global exposure and vulnerability towards natural hazards: the disaster risk index. Natural Hazards Earth SystSci 9(4):1149-1159

Reasenberg PA, Jones LM (1994) Earthquake aftershocks: update. Science 265(5176):1251-1252

Soni S, Pirkul H (2002) Design of survivable networks with connectivity requirements. Telecommun Syst 20(1):133-149

Sörensen K (2007) Distance measures based on the edit distance for permutation-type representations. J Heuristics 13(1):35-47

Tiede D, Hoffmann C, Füreder P, Hölbling D, Lang S (2010) Automated damage assessment for rapid geospatial reporting-first experiences from the Haiti earthquake 2010. In: Car A, Griesebner G, Strobl J (eds) Geospatial Crossroads @ GI Forum'10. Proceedings of the Geoinformatics Forum Salzburg, pp 207-210

Van Wassenhove LN (2006) Humanitarian aid logistics: supply chain management in high gear. J Oper Res Soc 57(5):475-489

Viswanath K, Peeta S (2003) The multicommodity maximal covering network design problem for planning critical routes for earthquake response. 82nd Annual meeting of the transportation research board

Yi W, Özdamar L (2007) A dynamic logistics coordination model for evacuation and support in disaster response activities. Eur J Oper Res 179:1177-1193

Zitzler E, Thiele L (1999) Multiple objective evolutionary algorithms: a comparative case study and the strength of the pareto approach. IEEE Trans Evolut Comput 3(4):257-271

Zografos KG, Androutsopoulos KN (2008) A decision support system for integrated hazardous materials routing and emergency response decisions. Transp Res Part C: Emerg Technol 16(6):684-703 